\section{Fully protected MPAs can reduce patchiness of target species}

Marine protected areas (MPAs) are often used to protect patches of habitat that support species targeted for protection. Ideally, these protected habits should be well-connected so that protected species can flow from one MPA to its neighbors. $\beta$-diversity has been proposed as a way to measure this connectivity by examining the distribution of species within a defined area. High $\beta$-diversity indicates more species diversity and a more uniform spatial distribution of species, making these areas more resilient. Low $\beta$-diversity can indicate that species are distributed in a more "patchy" manner or where communities are dominated by just a few species.

The authors studied Italy's Punta Campanella MPA in the Tyrrhenian Sea. The 1300-ha MPA is only $0.7 \mathrm{~nm}$ from the coast and prone to anthropogenic pressures. It features a small, 21-ha no-take zone surrounded by a relatively unprotected area that allows fishing and other human activities. A visual census of species was taken from one site within this no-take area, and six sites in the surrounding zone between June and October 2008. Fish assemblages were measured at $5 \mathrm{~m}, 10 \mathrm{~m}$, and $20 \mathrm{~m}$ depths.

Statistical analysis showed no geographic differences in data (fish biomass and abundance) among the six unprotected sites, allowing comparisons to be made as a group between these sites and the fully protected site. The fully protected site was found to have high $\beta$-diversity: fish assemblages changed at depth to reflect a different makeup of species. Species richness, total abundance, and total biomass were all higher at each depth than in the unprotected sites. In the unprotected sites, fish assemblages were more "patchy" and exhibited low $\beta$-diversity. Plotting abundance-biomass curves for the fully protected and unprotected sites indicated that fishing pressure impacted the community by removing large predators of target populations. Non-target fish were seemingly unaffected by protection, indicating there is no meaningful difference in habitat quality complicating comparisons between sites.

The low $\beta$-diversity of unprotected MPAs likely reduces their ecological connectivity: small patches of fish are less likely to flow from one MPA to the next. Thus, there is less resilience associated with these unprotected MPAs. The authors illustrate yet again that fully protected MPAs provide the most ecological benefits.

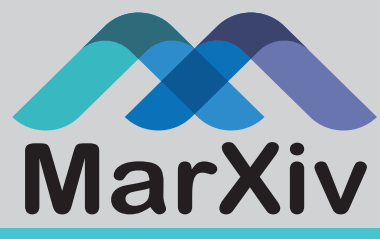

This is a summary of: Does full protection count for the maintenance of $\beta$-diversity patterns in marine communities? Evidence from Mediterranean fish assemblages

Accessible at: https://marxiv.org/ts9a4

Authors:

Luca Appolloni, Stanislao Bevilacqua, Luisa Sbrescia, Roberto Sandulli, Antonio

Terlizzi, Giovanni Russo

Added to MarXiv:

April 2018

Published: Aquatic Conservation: Marine and Freshwater Ecosystems, 2018

Suggested Citation: Fully protected MPAs can reduce patchiness of target species. OCTO (2018). DOI: 10.17605/OSF.IO/XWUHS

See more MarXiv summaries at https://www.marxivinfo.org/ summaries

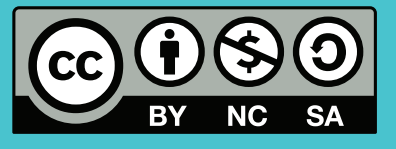

MarXiv is an ОСТO Initiative
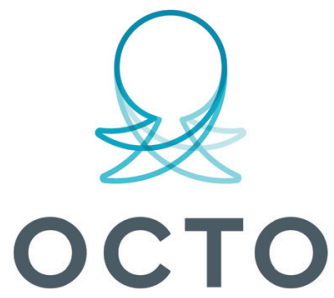

OPEN COMMUNICATIONS FOR THE OCEAN 